\title{
PHOTOTHERMAL INTERFEROMETRY FOR NONDESTRUCTIVE SUBSURFACE DEFECT DETECTION
}

\section{Z. SODNIK and H.J. TIZIANI}

Institut für Technische Optik, Universität Stuttgart, Pfaffenwaldring 9, D-7000 Stuttgart 80, Fed. Rep. Germany

Received 6 January 1986; revised manuscript received 5 March 1986

\begin{abstract}
A symmetrical interferometer as thermal expansion detector was developed for photothermal nondestructive material analysis. Phase and amplitude exchange phenomena have been observed, when introducing an electronic reference phase shifter mixed to the interferometer signal. Experimental and theoretical results obtained with this system are presented.
\end{abstract}

\section{Introduction}

For nondestructive material analysis many photo. acoustic [1-5] and some photothermal [6-8] experiments have been published in the last years. Few publications have been made in the field of photothermal interferometry $[9,10]$.

Even the name is confusing, because in photothermal interferometry the thermal expansion of a specimen is measured rather than interferometry done with thermal waves.

A photothermal process is the absorption of modulated light that modulates the surface temperature of a specimen and causes a heat diffusion called thermal wave to propagate through the material. The thermal wave is reflected at subsurface defects and leads to thermal expansions that are interferometrically detected.

This technique is very sensitive [11] but critical to mechanical vibration. For this reason a special symmetrical interferometer is used (fig. 1).

Measuring the amplitude and phase of the interferometer signal with a lock-in-amplifier leads to un. expected results in case of mixing a fraction of the reference signal to the interferometer signal.

The aim of the paper is to present a system for nondestructive detection of subsurface defects using reflected thermal waves. In contrary to other publications concerning photothermal experiments our system is equal sensitive if measuring phase response or amplitude.

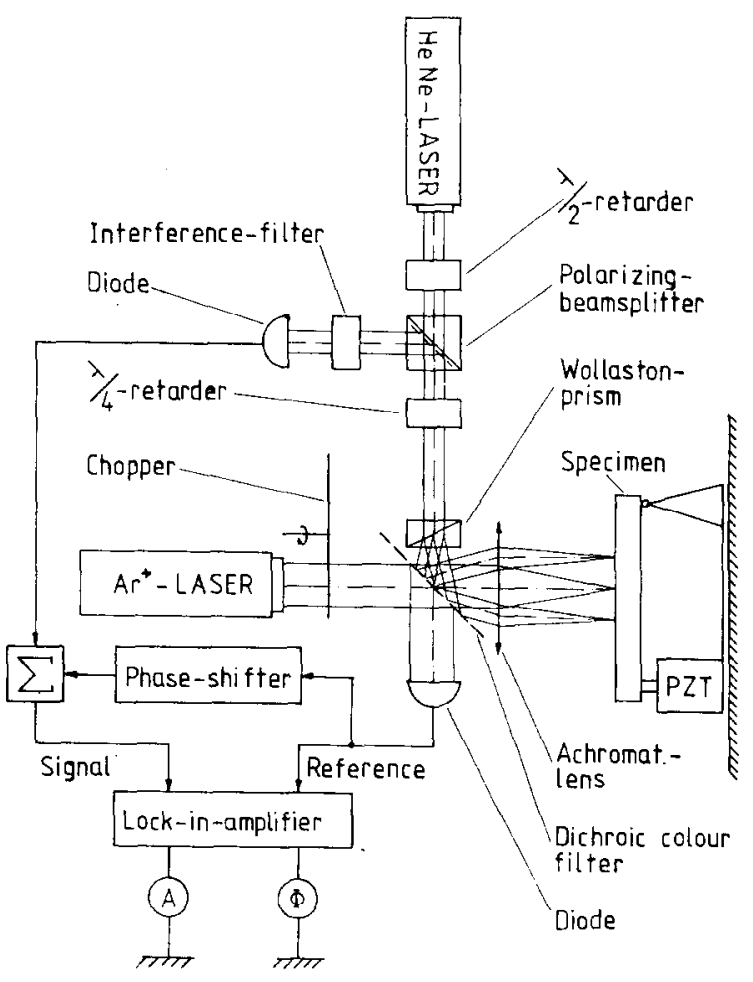

Fig. 1. The experimental arrangement. 


\section{Experimental arrangement}

\subsection{Interferometer}

An interferometer to minimize the influence of vibrations has been built. A similar interferometer was described in 1954 by Nomarski [12] and later used for testing mirror surfaces for X-ray optics [13].

The polarisation of a $\mathrm{HeNe}$-laser beam as shown in fig. 1 is rotated by a $\lambda / 2$-plate to achieve maximum transmittance of the polarizing beam splitter. The following $\lambda / 4$-plate leads to circular polarization which is separated in two orthogonal polarized beams by a Wollaston prism.

These two beams are reflected by a dichroic colour filter and focused onto the specimen by an achromatic lens. After reflection they are recombined on their way back in the Wollaston prism. Passing through the $\lambda / 4$-plate again leads to maximum reflectance of the polarization beam splitter at 45 degrees with respect to the recombined orthogonal polarized beams.

The zero order interference fringe is detected by a photodiode. A narrowband interference filter with the maximum transmittance at $633 \mathrm{~nm}$ in front of the diode stops the scattered light of the argon laser.

The adjustment of the interferometer is simply done by tilting the specimen with a computer controlled piezo crystal.

The distance of the two HeNe-laser spots on the specimen was optimized to achieve maximum signal by asymmetrical thermal expansion of the surface.

\subsection{Thermal wave generating system}

The thermal wave is generated by periodical heating of the specimen's surface using a $500 \mathrm{~mW}$ argon laser at $488 \mathrm{~nm}$. The laser beam is chopped at frequencies between 10 and $250 \mathrm{~Hz}$ and focussed, after passing through a dichroic colour filter, onto the surface exactly in between the two HeNe-laser spots. The surface wave, that affects both HeNe-laser spots in the same manner, can not be detected by symmetrical arranged interferometer.

\subsection{Phase shifter}

Measuring the phase shift between the argon-laser beam and the interferometer signal by a lock-in-am- plfier leads to trouble in case of perfect symmetry of the specimen within the detection radius, with respect to the HeNe-laser spots. In that case the amplitude is zero and therefore the phase measurement gives meaningless stochastic results.

Therefore a part of the reference signal is electrical mixed to the interferometer signal, just big enough to stabilize the lock-in-amplifier. Since this injection signal is digital it can easily be phase shifted.

\section{Theory}

\subsection{Thermal wave propagation}

Heat conduction in solids is a diffusion phenomenon which can be described for the one dimensional case by [14]

$\partial^{2} T(x, t) / \partial x^{2}-D^{-1} \partial T(x, t) / \partial t=0$,

with the thermal propagation length and time dependent temperature $T(x, t)$ and with the thermal diffusivity

$D=x / \rho c$,

which depends on the thermal conductivity $x$, the density $\rho$ and the heat capacity $c$.

For sinusoidal heating the solution is given by

$T(x, t)=T_{0} \exp (-x / \mu) \sin (\omega t-x / \mu)$,

with the thermal diffusion length

$\mu=(D / \pi \nu)^{1 / 2}$

which depends on the thermal diffusivity $D$ and the heating frequency $\nu$. The thermal wavelength $\lambda_{t}$ is given by

$\lambda_{\mathrm{t}}=2 \pi \mu$.

Fig. 2 shows the principle for the detection of a subsurface hole using a symmetric differential expansion detector with two HeNe-laser beams, a centered argonlaser beam for heat generation and two reflected thermal waves of interest.

The one dimensional solution proved to be a good model of the three dimensional system if eq. (3) is multiplied with a thermal propagation length $x$-dependent damping factor. Best results were obtained when dividing eq. (3) with the square root of $x$. 


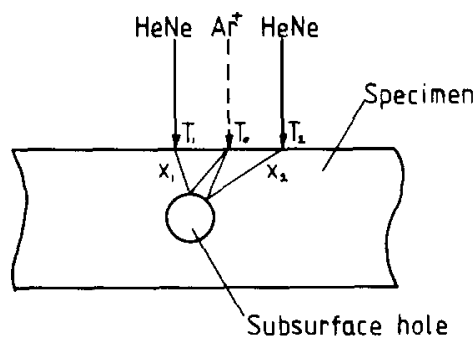

Fig. 2. The thermal wave detection principle.

The temperatures $T_{1}$ and $T_{2}$ are then given by

$T_{i}=\left(T_{0} / x_{i}\right) \exp \left(-x_{i} / \mu\right) \sin \left(\omega t-x_{i} / \mu\right)$,

$i=1,2$.

The optical path difference $\delta$ in the interferometer is approximately proportional to $\left(T_{1}-T_{2}\right)$.

\subsection{Interferometer adjustment}

The intensity $I_{\mathrm{s}}$ at the photodiode is given by

$I_{\mathrm{s}}=\frac{I_{\mathrm{max}}+I_{\mathrm{min}}}{2}+\frac{I_{\mathrm{max}}-I_{\mathrm{min}}}{2} \cos \left(4 \pi \delta / \lambda_{\mathrm{s}}\right)$,

with the maximum intensity $I_{\mathrm{max}}$, the minimum intensity $I_{\mathrm{m} \text { in }}$ and the HeNe-wavelength $\lambda_{\mathrm{s}}$.

The optical path difference $\delta$ is given by

$\delta=\delta_{\mathrm{t}}+\delta_{\mathrm{r}}+\delta_{\mathrm{p}}$,

with the thermal expansion $\delta_{t}$, the specimen's surface roughness $\delta_{\mathrm{r}}$ and the piezo crystal displacement $\delta_{\mathrm{p}}$. by

The computer control of the piezo crystal is given

$\delta_{\mathrm{p}}=\frac{3}{8} \lambda_{\mathrm{s}}-\delta_{\mathrm{r}}$

which compensates the surface roughness and adjusts the interferometer to work in the linear region with maximum positive gradient. This is important for maximum sensitivity and minimum adjustment time of the lock-in-amplifier. This also changes the cosine in eq. (7) into a sine, and because the thermal expansions are small in comparison to the wavelength $\lambda_{s}$, eq. (7) can be written

$I_{\mathrm{s}}=\frac{I_{\mathrm{max}}+I_{\min }}{2}+\left(I_{\max }-I_{\min }\right) \frac{2 \pi}{\lambda_{\mathrm{s}}} \delta_{\mathrm{t}}$.

\subsection{Detection signal}

The injection signal $I_{\mathrm{I}}$ from the phase-shifter is

$I_{\mathrm{r}}=I_{0} \sin \left(\omega t-\phi_{0}\right)$

with the reference amplitude $I_{0}$ and the reference phase shift $\phi_{0}$. Finally the signal under investigation is

$I=I_{\mathrm{s}}+I_{\mathrm{r}}$,

which is fed to the lock-in-amplifier.

\section{Results}

A prefabricated aluminum test sample (fig. 3e) with a subsurface hole of $0.8 \mathrm{~mm}$ diameter and $0.4 \mathrm{~mm}$ depth was examined at a modulation frequency of $140 \mathrm{~Hz}$ and $12 \mathrm{mV}$ injection voltage phase shifted in four steps between $0^{\circ}$ and $-135^{\circ}$.

Both phase and amplitude can have equivalent information about the subsurface structure. That is shown by comparing the zero and the $-90^{\circ}$ phase shift measurements (fig. 3a and fig. 3c) respectively. The $-90^{\circ}$ measurement's phase is equal to the $0^{\circ}$ amplitude and the $-90^{\circ}$ amplitude is the inverse zero degree's phase.

This happens when a fraction of the reference signal, phase shifted by $-90^{\circ}$, is mixed (injected) to the interferometer signal. This is manifested by comparing fig. $3 \mathrm{~b}$ and fig. $3 \mathrm{~d}$. The theoretical results are overlayed as dashed lines in the diagrams (figs. 3a-d).

It should be noted, that the measurement system added $180^{\circ}$ to the phase results in fig. $3 \mathrm{c}$ and fig. $3 \mathrm{~d}$. The mean values of all plots correspond to the injection phase (with an offset) and the injection voltage respectively.

These four measurements were obtained one after the other scanning the same line on the specimen. They show very low noise, because the peaks in all plots mostly correspond to their $-90^{\circ}$ phase injected counterparts. This proves very high sensitivity to the surface parameters of the specinen.

By contrast to some publications [15] where it is pointed out, that the phase information is twice as sensitive as the amplitude information, we found in the described technique both to the equal.

No lock-in-amplifier with phase option is needed 


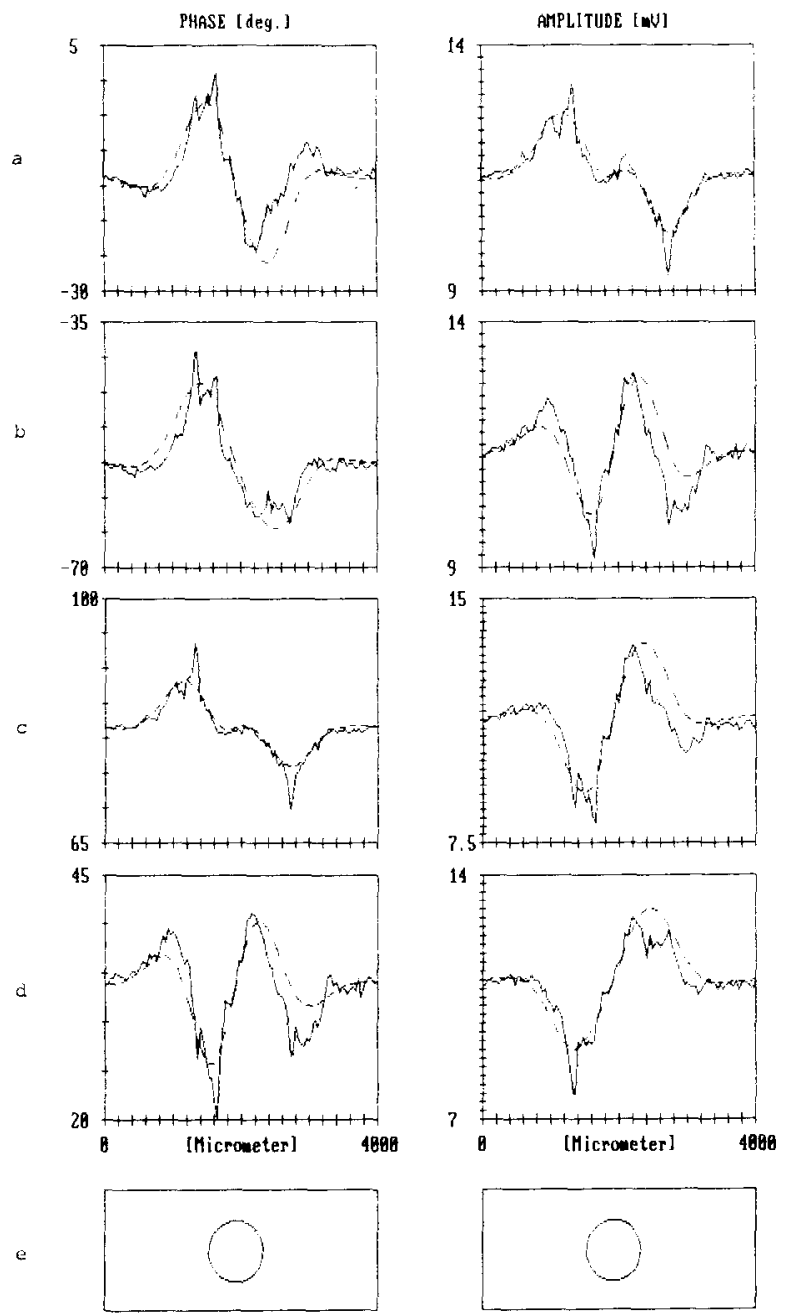

Fig. 3. Experimental and theoretical results obtained at 140 $\mathrm{Hz}$ modulation frequency. The diagrams show the scanning distance along the specimen versus phase response and amplitude respectively. The injection voltage is $12 \mathrm{mV}$ phase shifted by (a) $0^{\circ}$, (b) $-45^{\circ}$, (c) $-90^{\circ}$, (d) $-135^{\circ}$. (e) The specimen, an Al-plate with a prefabricated hole of $0.8 \mathrm{~mm}$ diametcr and $0.4 \mathrm{~mm}$ depth, shown at appropriate scale.

for phase response measurements. A simple vector voltmeter can be used by mixing the interferometer signal with the reference signal of appropriate amplitude and $90^{\circ}$ phase shift.

From the knowledge of the specimen's geometry and one of the results (phase response or amplitude), the other one can be calculated.

It should be possible to calculate the geometry of an unknown specimen with the knowledge of phase response and amplitude.

\section{Conclusion}

Indirect thermal wave detection by a symmetrical interferometer arrangement and an injection reference voltage mixed to the interferometer signal can be a powerful tool for nondestructive material analysis.

- Subsurface defects as small as $0.1 \mathrm{~mm}$ diameter and as deep as $1 \mathrm{~mm}$ below the surface can be detected with this technique. For higher resolution the modulation frequency must be increased, leading to a reduced thermal penetration depth and detection radius. With frequencies of several thousand Hertz resolutions in the order of micrometers can be achieved.

- Phase response and amplitude measurements can easily be done with a vector voltmeter and a $90^{\circ}$ phase shifter.

- It should be possible to calculate the geometry of an unknown specimen by knowledge of phase response and amplitude.

\section{Acknowledgement}

The authors would like to thank Dr. G. Busse for many helpful discussions.

\section{References}

[1] A. Rosencwaig, Photoacoustic and photoacoustic spectroscopy (John Wiley, 1980).

[2] A. Rosencwaig and A. Gersho, J. Appl. Phys. 47 (1976) 64.

[3] Y.H. Wong, R.L. Thomas and J.J. Pouch, Appl. Phys. Lett. 35 (1979) 368.

[4] H.J. Inglehard, K.R. Grice, L.D. Favro, P.K. Kuo and R.L. Thomas, Appl. Phys. Lett. 43 (1983) 446.

[5] C.R. Petts and H.K. Wickramashinge, Proc. IEEE Ultrasonic Symposium (1981) p. 832.

[6] P.E. Nordal and S.O. Kanstad, Physica Scripta 20 (1979) 659.

[7] G. Busse and P. Eyerer, Appl. Phys. Lett. 43 (1983) 355.

[8] G. Busse, Appl. Optics 21 (1982) 107.

[9] S. Ameri, E.A. Ash, V. Neuman and C.R. Petts, Electric. Lett. 17 (1981) 337. 
110] H. Dersch and N.M. Amer, contribution to 4 th Intern. Topical Meeting on Photoacoustic, thermal and related sciences (1985).

[11] Z. Sodnik and H.J. Tiziani, contribution to 4 th Intern. Topical Meeting on Photoacoustic, thermal and related sciences (1985).
[12] G. Normarski, J. Phys. Radium 16 (1955) $31 \mathrm{~S}$.

[13] G. Makosch and B. Solf, SPIE 316 (1981) 42.

[14] H. Ermert, F.H. Dacol and R.L. Melcher, contribution to 3rd European Conf. on Nondestructive testing (1984).

[15] G. Busse, Appl. Phys. Lett. 35 (1979) 759. 